\title{
HEPARIN AND NONANTICOAGULANT HEPARIN PRESERVE REGIONAL MYOCARDIAL CONTRACTILITY AFTER ISCHEMIA-REPERFUSION INJURY: ROLE OF NITRIC OXIDE
}

Peter C. Kouretas, $\mathrm{MD}, \mathrm{PhD}^{\mathrm{a}}$ Adam K. Myers, $\mathrm{PhD}^{\mathrm{b}}$

Young D. Kim, $\mathrm{MD}^{\mathrm{c}}$

Paul A. Cahill, $\mathrm{PhD}^{\mathrm{a}}$

Jeff L. Myers, MD, PhD

Yi-Ning Wang, MD ${ }^{\mathrm{a}}$

James V. Sitzmann, MD ${ }^{a}$

Robert B. Wallace, MD $^{\mathrm{a}}$

Robert L. Hannan, MD
Objectives: These studies were performed to determine the effect of heparin and nonanticoagulant heparin on myocardial function after ischemiareperfusion and to further evaluate the role that the nitric oxide-cyclic guanosine monophosphate pathway plays in mediating the effect of heparin. Methods: Fifteen dogs were subjected to 15 minutes ischemia followed by 120 minutes reperfusion and pretreated with either saline solution, bovine heparin $(6.0 \mathrm{mg} / \mathrm{kg}$ intravenously), or $N$-acetyl heparin $(6.0 \mathrm{mg} / \mathrm{kg}$ intravenously), a heparin derivative without anticoagulant properties. The left anterior descending artery was occluded for 15 minutes and regional systolic shortening, a unitless measure of myocardial contractility, assessed during reperfusion. To evaluate the role of nitric oxide, the inhibitor $N^{\omega}$-nitro-L-arginine, $1.5 \mathrm{mg} / \mathrm{kg}$ intracoronary, was given before heparin administration. Myocardial levels of cyclic guanosine monophosphate, the second messenger of nitric oxide, were also measured in the $\mathrm{N}$-acetyl heparin group using radioimmunoassay. Results: Regional systolic shortening was significantly decreased in the saline group during 60 and 120 minutes compared with before ischemia $(9.2 \pm 1.0$ and $9.0 \pm 0.9$ vs $12.2 \pm$ 1.2, $p \leq 0.0003$ ). Heparin and $N$-acetyl heparin-treated dogs, however, showed preservation of systolic shortening throughout reperfusion. Administration of nitro-L-arginine significantly attenuated the protective effect of heparin $(9.2 \pm 1.2$ vs $12.7 \pm 1.1, p \leq 0.0001)$ and $N$-acetyl heparin $(9.3 \pm$ 0.3 vs $12.8 \pm 0.4, p \leq 0.0001)$ during 120 minutes reperfusion. Myocardial levels of cyclic guanosine monophosphate were also significantly increased in the $N$-acetyl heparin group compared with saline $(199.1 \pm 7.1$ vs $103.5 \pm$ $4.5 \mathrm{pmol} / \mathrm{mg}, p \leq 0.0001)$. Conclusions: Heparin preserves myocardial contractility after ischemia-reperfusion independent of its anticoagulant properties. Furthermore, the protective effects of heparin during ischemiareperfusion are mediated, at least in part, through a nitric oxide-cyclic guanosine monophosphate pathway. (J Thorac Cardiovasc Surg 1998;115: 440-9)
From the Departments of Surgery, ${ }^{\text {a }}$ Physiology and Biophysics, ${ }^{\text {b }}$ and Anesthesiology, ${ }^{\mathrm{c}}$ and Division of Thoracic and Cardiovascular Surgery, ${ }^{\mathrm{d}}$ Georgetown University Medical Center, Washington, D.C. and University of Virginia Health Sciences Center, Charlottesville, Va.

This work was supported by an American Heart Association grant RX4351-676 to R.L.H.

Read at the Seventy-seventh Annual Meeting of The American Association for Thoracic Surgery, Washington, D.C., May 4-7, 1997.

Received for publication May 6, 1997; revisions requested June 25, 1997; revisions received Sept. 26, 1997; accepted for publication Oct. 8, 1997.

Address for reprints: Robert L. Hannan, MD, University of Virginia Health Sciences Center, Department of Surgery, Box 3501, Charlottesville, VA 22908.

Copyright (C) 1998 by Mosby, Inc.

$0022-5223 / 98 \$ 5.00+0 \quad \mathbf{1 2 / 6 / 8 6 8 8 7}$
$B$ rief episodes of myocardial ischemia followed by reperfusion are common clinical events in patients with coronary artery disease. These events result in episodes of coronary endothelial and regional myocardial dysfunction. ${ }^{1}$ This endothelial dysfunction results in an impaired ability of the endothelium to synthesize and release nitric oxide (NO). ${ }^{2}$ Recent reports indicate an integral role for NO released from both the endothelium of the vasculature and the endocardium in the maintenance of myocardial contractility. ${ }^{3,4}$ Furthermore, inhibition of NO synthesis has been demonstrated to increase myocardial dysfunction after ischemiareperfusion (IR). ${ }^{5}$ These data indicate a critical role for NO in both normal myocardial function and in 
the maintenance of myocardial function in the setting of IR injury.

Pharmacologic preservation of myocardial function in the setting of IR injury remains a clinical challenge. Heparin is widely used clinically as an anticoagulant and has been demonstrated to protect the myocardium in the setting of IR injury. ${ }^{6}$ Recent reports have demonstrated increased levels of nitrite and nitrate, the metabolites of $\mathrm{NO},{ }^{7}$ after heparin administration in vivo. In the setting of myocardial IR injury, preischemic administration of heparin and heparin derivatives diminishes the extent of myocardial infarct size. ${ }^{8}$

The rationale for this study stems from the observation that discontinuation or reversal of heparin in patients at risk for myocardial ischemia after cardiac catheterization or cardiopulmonary bypass, for example, was occasionally associated with disastrous myocardial dysfunction. We therefore hypothesized that heparin may be regulating myocardial contractile function possibly independent of its anticoagulant activity. We also hypothesized that the effect of heparin may be mediated, in part, through modulation of the NO pathway. Therefore the initial aims of these studies were to establish the effects of heparin and nonanticoagulant heparin on regional myocardial function preservation in the setting of brief IR injury. Subsequent studies were then performed to explain the potential role of a heparinendothelial-derived NO pathway in the prevention of myocardial dysfunction associated with IR injury.

\section{Material and methods}

Animals. Male mongrel dogs ( 25 to $35 \mathrm{~kg}$ ) were used in these studies. All experiments were approved by the Georgetown Animal Care and Use Committee (GUACUC) and conformed to the guidelines of the "Guide for the Care and Use of Laboratory Animals" prepared by the National Institutes of Health $(\mathrm{NIH}$ Publication No. 85-23, revised 1985).

Materials: Bovine heparin, $N$-acetyl heparin and $N^{\omega}$ nitro-L-arginine (L-NNA) were purchased from Sigma Chemical (St. Louis, Mo.).

Surgical preparation. Animals were fasted overnight before the operation with water available ad libitum. Anesthesia was induced with sodium thiopental $(25 \mathrm{mg} /$ $\mathrm{kg}$, intravenous) and after endotracheal intubation the lungs were mechanically ventilated. Anesthesia was maintained with halothane $(1.5 \%)$ for the remainder of the experimental period. Pancuronium bromide $(0.1 \mathrm{mg} / \mathrm{kg}$, intravenous) was also administered to produce complete muscle relaxation. Surgical preparation and collection of data were performed at an inspired oxygen fraction of 1.0 and a respiratory rate of 10 to 13 ventilations/min. Endtidal halothane, carbon dioxide, and arterial oxygen satu- ration were continuously monitored with a POET agent analyzer equipped with a pulse oximeter (Criticare Systems, Waukesa, Wis.). End-tidal carbon dioxide levels were maintained between 33 and $37 \mathrm{~mm} \mathrm{Hg}$ and end-tidal halothane was maintained at $1.5 \%$ throughout the study. Body temperature was maintained in the normothermic range with a warming blanket.

A left thoracotomy was performed in the fifth intercostal space and the heart was suspended in a pericardial cradle. The left anterior descending (LAD) artery was then exposed and dissected free of the myocardium immediately distal to the first major diagonal branch. A $2.0 \mathrm{~mm}$ ultrasonic flow probe (Transonic Systems, Ithaca, N.Y.) was positioned around the LAD artery distal to the first diagonal for the continuous monitoring of LAD artery flow with a Transonic T20 two-channel ultrasonic blood flowmeter. A rubber vessel loop was then placed around the LAD artery at this site and the free ends passed through a narrow silicone rubber tube for use as a snare to occlude the LAD artery.

A polyvinyl chloride catheter (Deseret Medical Inc., Sandy, Utah) was placed in the right femoral artery. A high-fidelity pressure transducer (Millar Instruments, Inc., Houston, Tex.) was then placed through this catheter into the aortic arch for measurement of aortic pressure. A second transducer was placed in the left ventricle for the continuous monitoring of left ventricular pressure. A pair of ultrasonic dimension transducers (Vernitron, Bedford, Ohio) were then implanted 10 to $12 \mathrm{~mm}$ apart in the inner one third of the subendocardium of a region of the anterior free wall of the left ventricle supplied by the LAD artery to measure regional systolic shortening, a measure of myocardial contractility. ${ }^{9}$ All animals were allowed at least 15 minutes to stabilize after surgical instrumentation.

\section{Experimental protocol}

In vivo studies. Thirty animals were randomly assigned to one of six treatment groups: saline, bovine heparin, $N$-acetyl heparin, the NO synthase (NOS) inhibitor $N^{\omega}$ nitro-L-arginine (L-NNA), L-NNA and bovine heparin, and L-NNA and $N$-acetyl heparin. The treatment was administered after instrumentation and before LAD artery occlusion. The dose of bovine heparin was determined on the basis of heparin dose-response curves performed for each animal using the Hepcon HMS microprocessor (Medtronic HemoTec, Inc., Englewood, Colo.). The dose of bovine heparin needed for each individual dog was based on the endogenous level of heparin in each animal and presented as a heparin level $(\mathrm{mg} / \mathrm{kg})$ that was needed to achieve adequate anticoagulation. The end point of our dosing was the circulating heparin level needed to achieve adequate anticoagulation. Heparin levels were checked every 15 minutes using the Hepcon microprocessor and an appropriate level of heparin was infused as a bolus to maintain the optimum heparin level. The average dose of bovine heparin administered over the course of the protocol for all dogs was 6.0 $\mathrm{mg} / \mathrm{kg}$. Therefore the dose of $N$-acetyl heparin chosen was also $6.0 \mathrm{mg} / \mathrm{kg}$. This dose of $N$-acetyl heparin is also consistent with the total dose administered in a similar canine model of myocardial IR injury. ${ }^{8}$ The dose of 
L-NNA $(1.5 \mathrm{mg} / \mathrm{kg})$ was administered intracoronary to selectively inhibit the NO pathway in the region of ischemia and to avoid the hemodynamic complications of increased arterial and left ventricular pressure associated with systemic administration consistent with previous experiments performed by Duncker and Bache ${ }^{10}$ in a similar canine model. L-NNA was administered 30 minutes before heparin and $N$-acetyl heparin. Activated clotting times and partial thromboplastin time were also measured as an index of the degree of anticoagulation.

After treatment with either saline solution, bovine heparin, or $N$-acetyl heparin, animals were subjected to 15 minutes of regional ischemia by LAD artery occlusion followed by 120 minutes of reperfusion. Cessation of LAD flow, wall motion changes, cyanosis of the anterior wall, and typical electrocardiographic changes immediately after LAD artery occlusion were used as indices of successful anterior wall ischemia. Hemodynamic measurements, including regional systolic shortening, were recorded before ischemia and at 15, 60, and 120 minutes after reperfusion.

Data collection and analysis. The signals from the pressure transducers, ultrasonic flow probes, and sonomicrometry crystals were electronically processed by use of a calibration control unit (Millar Instruments) and a Gould transducer (model 13; Gould, Inc., Cleveland, Ohio). The analog signals were subsequently amplified and converted to digital signals at a sampling rate of $200 \mathrm{~Hz}$. Analog signals were also displayed on an oscilloscope (model 5B10; Tektronic, Beaverton, Ore.) for signal verification throughout the experimental procedure. After experimentation, stored signals were digitized and the data transferred for hemodynamic computations. Approximately 10 heartbeats were analyzed per intervention. Regional systolic shortening, a unitless measure of myocardial contractility, was determined using the following equation:

$$
\frac{\text { End-diastolic length }- \text { End-systolic length }}{\text { End-diastolic length }} \times 100
$$

where end-diastolic length is measured at the onset of the left ventricular isovolumic contraction and end-systolic length is measured at the maximum negative rate of pressure rise $(\mathrm{dP} / \mathrm{dt})$. After the completion of the in vivo experiments, the left ventricular myocardium in the distribution of the LAD artery was harvested in animals exposed to saline or $N$-acetyl heparin, snap frozen in liquid nitrogen, and stored at $-80^{\circ} \mathrm{C}$ for cyclic guanosine monophosphate (cGMP) analysis.

\section{In vitro studies}

cGMP levels in myocardium. Left ventricular myocardium from ischemic and nonischemic regions was placed in $0.1 \mathrm{~mol} / \mathrm{L}$ hydrochloric acid and homogenized in a Tissumiser (Tekmar, Cincinnati, Ohio). The homogenate was then placed on ice for 30 minutes to extract cyclic nucleotides. The homogenate was then centrifuged at $6500 \mathrm{~g}$ for 30 minutes at $4^{\circ} \mathrm{C}$ and the resulting cytosolic fraction analyzed for cGMP levels by means of radioimmunoassay. ${ }^{11}$ In brief, cGMP standards $(1 \mu \mathrm{mol} / \mathrm{L})$ and experimental samples were acetylated using an acetic anhydride/triethylamine solution (1:2). A standard curve was then generated using serial dilutions of $1 \mu \mathrm{mol} / \mathrm{L}$
cGMP. cGMP antiserum (1:3,000 dilution) and $\left[{ }^{125} \mathrm{I}\right] \mathrm{cGMP}$ was then added to both the standards and unknowns and incubated for a further 16 hours at $4^{\circ} \mathrm{C}$. The assay was stopped by the addition of $100 \mu \mathrm{l}$ of $1 \%$ $\gamma$-globulin and $2 \mathrm{ml} 25 \%$ polyethylene glycol. The samples were then placed in a vortex before centrifugation at $7500 \mathrm{~g}$ for 30 minutes at $4^{\circ} \mathrm{C}$. The supernatant was then aspirated and the pellet counted using a scintillation counter. Myocardial cGMP levels were normalized to protein content with serum albumin as a standard and expressed in picomoles per milligram of myocardial protein.

To further explain the role of the NO-cGMP pathway on the $N$-acetyl heparin-mediated effect, cGMP levels were measured after 15 minutes ischemia and 120 minutes reperfusion in a separate group of dogs $(n=5)$ pretreated with the NOS inhibitor L-NNA at the same intracoronary dose of $1.5 \mathrm{mg} / \mathrm{kg}$.

Coagulation parameters. Activated clotting times were measured immediately before and 60 minutes after heparin and $N$-acetyl heparin administration using a Hepcon HMS microprocessor (Medtronic HemoTec). Blood samples were also assayed for the measurement of partial thromboplastin time (Vet Research, Farmingdale, N.Y.).

Statistical analysis. Myocardial contractility during reperfusion was compared with preischemic values within each treatment group with a two-way analysis of variance for repeated measures. Values after treatment with heparin and $N$-acetyl heparin were compared with saline control using a two-tailed unpaired Student's $t$ test. Myocardial levels of cGMP after treatment with $N$-acetyl heparin before and after IR injury were compared in a similar manner. Measurements are reported as mean values \pm the standard error of the mean.

\section{Results}

Myocardial contractility. Regional systolic shortening was significantly decreased in animals subjected to IR injury alone during 60 and 120 minutes reperfusion ( $p<0.0003$, Fig. 1$)$. Groups that were treated with bovine heparin and $N$-acetyl heparin displayed preservation of regional systolic shortening throughout the entire period of reperfusion (Fig. $1)$.

NO inhibition. Intracoronary L-NNA did not significantly increase hemodynamic parameters, which are affected by systemic inhibition of NO (Table I). LAD artery flow was also not significantly altered after regional infusion of L-NNA (Table I). Regional inhibition of NO with L-NNA caused a significant decrease in regional systolic shortening during 60 and 120 minutes of reperfusion compared with preischemia ( $p<0.0001$, Fig. $2, A$ and $B$ ). Inhibition of NOS before IR injury, however, did not cause a significant decrease in regional myocardial contractility during preischemia or during reperfusion compared with IR alone ( $p=$ not significant, Fig. $2, A$ 


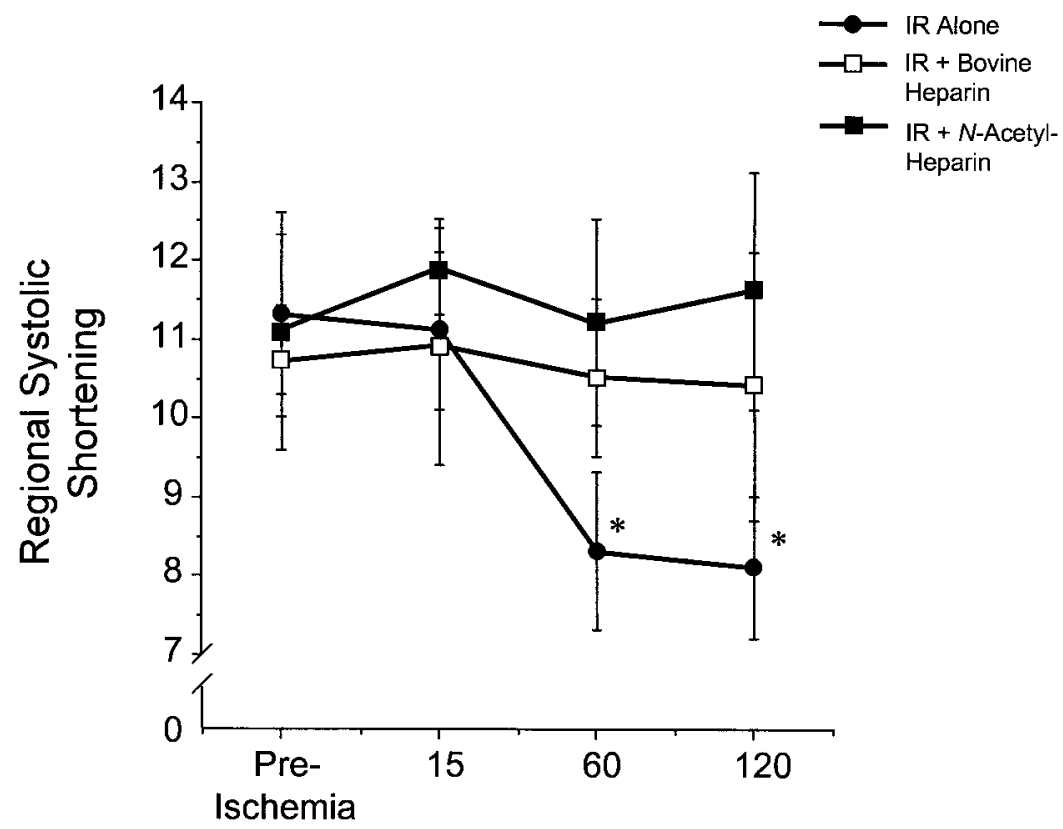

Minutes Reperfusion

Fig. 1. Regional systolic shortening during reperfusion after preischemic treatment with bovine heparin and $N$-acetyl heparin. Groups represent preischemic treatment with saline solution $(n=5)$, bovine heparin $(6.0 \mathrm{mg} / \mathrm{kg}$, intravenous, $n=5)$, and $N$-acetyl heparin $(6.0 \mathrm{mg} / \mathrm{kg}$, intravenous, $n=5)$. Data are expressed as means \pm the standard error of the mean. Asterisks indicate $p<0.0003$ versus preischemic value within each group. $I R$, Ischemia-reperfusion.

Table I. Hemodynamic profile before ischemia and treatment and during reperfusion in dogs treated with saline (1), the nitric oxide synthase inhibitor nitro-L-arginine (L-NNA) alone (2), L-NNA with bovine heparin (3), or L-NNA with the nonanticoagulant derivative, $N$-acetyl heparin (4)

\begin{tabular}{|c|c|c|c|c|c|c|c|c|}
\hline & & & & & \multicolumn{4}{|c|}{120 Minutes reperfusion, posttreatment } \\
\hline & \multicolumn{4}{|c|}{ Preischemia, pretreatment } & \multirow{2}{*}{$\begin{array}{c}1 \\
\text { Saline } \\
\text { alone }\end{array}$} & \multirow{2}{*}{$\begin{array}{c}2 \\
L-N N A \\
\text { alone }\end{array}$} & \multirow{2}{*}{$\begin{array}{c}3 \\
L-N N A+ \\
\text { heparin }\end{array}$} & \multirow{2}{*}{$\begin{array}{c}4 \\
L-N N A+ \\
N \text {-acetyl } \\
\text { heparin }\end{array}$} \\
\hline & 1 & 2 & 3 & 4 & & & & \\
\hline $\mathrm{HR}$ & $102.4 \pm 5.8$ & $102.7 \pm 4.3$ & $110.8 \pm 3.5$ & $94.5 \pm 4.6$ & $95.0 \pm 5.4$ & $98.0 \pm 2.1$ & $101.3 \pm 8.1$ & $86.3 \pm 4.8$ \\
\hline LAD flow & $16.0 \pm 0.8$ & $16.0 \pm 1.5$ & $18.2 \pm 2.4$ & $19.2 \pm 1.9$ & $15.0 \pm 1.0$ & $18.0 \pm 1.7$ & $17.0 \pm 2.7$ & $19.0 \pm 1.9$ \\
\hline MAP & $74.6 \pm 7.2$ & $75.2 \pm 1.6$ & $91.1 \pm 6.6$ & $91.3 \pm 6.8$ & $75.7 \pm 5.6$ & $80.7 \pm 3.1$ & $81.0 \pm 6.2$ & $86.2 \pm 8.4$ \\
\hline LVP systolic & $85.2 \pm 6.5$ & $85.0 \pm 1.4$ & $99.2 \pm 6.5$ & $106.6 \pm 6.6$ & $80.4 \pm 5.5$ & $87.8 \pm 3.6$ & $90.2 \pm 6.7$ & $97.2 \pm 7.4$ \\
\hline LVEDP & $10.8 \pm 2.7$ & $7.3 \pm 1.8$ & $11.2 \pm 1.3$ & $12.6 \pm 2.2$ & $7.5 \pm 2.9^{*}$ & $4.2 \pm 0.8^{*}$ & $6.8 \pm 0.8^{*}$ & $10.2 \pm 1.8^{*}$ \\
\hline RSS & $12.2 \pm 1.2$ & $11.5 \pm 0.4$ & $12.7 \pm 1.1$ & $12.8 \pm 0.4$ & $9.0 \pm 0.9^{*}$ & $8.8 \pm 0.5^{*}$ & $9.2 \pm 1.2^{*}$ & $9.3 \pm 0.3 *$ \\
\hline
\end{tabular}

Values presented as mean \pm standard error of the mean $H R$, Heart rate (beats/minute); LAD flow, left anterior descending artery flow (ml/min), MAP, mean arterial pressure $(\mathrm{mm} \mathrm{Hg}) ; L V P$, left ventricular pressure $(\mathrm{mm} \mathrm{Hg}) ; L V E D P$, left ventricular end-diastolic pressure $(\mathrm{mm} \mathrm{Hg}) ; R S S$, regional systolic shortening (unitless).

${ }^{*} p \leq 0.0003$ comparing reperfusion with preischemia.

and $B$ ). However, the protective effect of bovine heparin and $N$-acetyl heparin on regional myocardial function during 60 and 120 minutes reperfusion was abrogated after preadministration of L-NNA (Fig. 2, $A$ and $B$ ).
Myocardial levels of cGMP. Regional myocardial levels of cGMP, the second messenger of NO, were significantly decreased after 120 minutes reperfusion compared with dogs that received saline solution in the absence of IR injury (103.5 \pm 4.5 

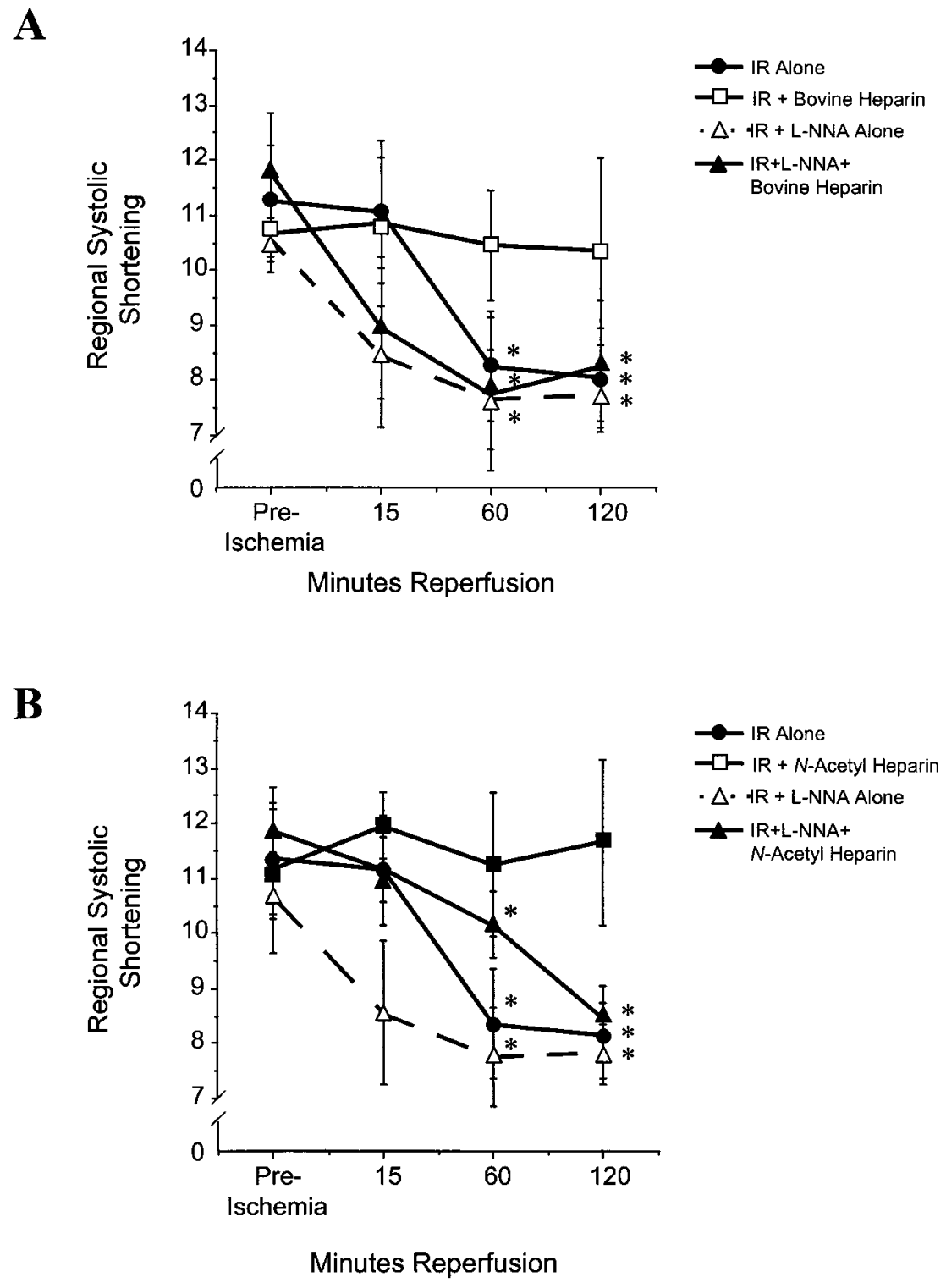

Fig. 2. Regional systolic shortening during 120 minutes of reperfusion after nitric oxide inhibition and treatment with bovine heparin (A) or $N$-acetyl heparin (B). Groups represent preischemic treatment with saline solution, bovine heparin, $N$-acetyl heparin, nitric oxide synthase inhibitor $N^{\omega}$-nitro-L-arginine $(L-N N A)$, L-NNA + bovine heparin, and L-NNA $+N$-acetyl heparin. There was an $n=5$ in each group. The dose of bovine heparin and $N$-acetyl heparin was $6.0 \mathrm{mg} / \mathrm{kg}$, intravenous. L-NNA $(1.5 \mathrm{mg} / \mathrm{kg})$ was administered in an intracoronary fashion before heparin and $N$-acetyl heparin. Data are expressed as means \pm the standard error of the mean. Asterisks indicate $p<0.0001$ versus preischemic value within each group. $I R$, Ischemia-reperfusion.

$\mathrm{pmol} / \mathrm{mg}$ vs $127.2 \pm 8.6 \mathrm{pmol} / \mathrm{mg}, p<0.04)$. After pretreatment with the nonanticoagulant heparin derivative $N$-acetyl heparin, myocardial levels of cGMP were increased compared with saline vehicle alone in the absence of IR injury $(127.2 \pm 8.6$ $\mathrm{pmol} / \mathrm{mg}$ vs $295.0 \pm 5.0 \mathrm{pmol} / \mathrm{mg}, p<0.0001$, Fig. 3 ,
A). In the setting of IR injury, pretreatment with $N$-acetyl heparin caused a significant increase in intramyocardial cGMP compared with IR alone $(103.5 \pm 4.5 \mathrm{pmol} / \mathrm{mg}$ vs $199.1 \pm 7.1 \mathrm{pmol} / \mathrm{mg}, p<$ 0.0001 , Fig. 3, $A$ ). Pretreatment with the NOS inhibitor L-NNA significantly attenuated the in- 
crease in cGMP in the left ventricular myocardium of groups treated with $N$-acetyl heparin in the setting of IR injury compared with IR alone and IR with $\mathrm{N}$-acetyl heparin pretreatment $(39.1 \pm 1.0$ $\mathrm{pmol} / \mathrm{mg}$ vs $103.5 \pm 4.5 \mathrm{pmol} / \mathrm{mg}$ and $199.1 \pm 7.1$ pmol/mg, $p<0.0001$, Fig. 3, B).

Coagulation profile. Activated clotting times and partial thromboplastin time were significantly increased above baseline in the bovine heparintreated groups $(p<0.05$, Table II). Treatment with $N$-acetyl heparin did not significantly alter any of the coagulation parameters studied, indicating that this heparin derivative was devoid of anticoagulant properties (Table II).

\section{Discussion}

Heparin binds to antithrombin III, accelerating the inhibition of thrombin and factor Xa resulting in anticoagulation. ${ }^{12}$ In addition to its ability to inhibit coagulation, it is now well established that heparin also modulates several other physiologic processes. We hypothesize a role for heparin in the preservation of myocardial function after IR injury, possibly through an NO-dependent pathway. The present data demonstrate, for the first time, that heparin preserves myocardial function in experimental IR injury by way of an NO-dependent pathway. Furthermore, these effects are independent of the anticoagulant properties of heparin because $\mathrm{N}$-acetyl heparin, which posses no anticoagulant activity, produced results similar to heparin.

One of the first physiologic processes that was shown to be modified by heparin was the inflammatory response. Heparin possesses several antiinflammatory properties, including the inhibition of polymorphonuclear leukocyte activation ${ }^{13}$ and inhibition of complement activation. ${ }^{14}$ More recently, studies have demonstrated that heparin modulates the release of numerous vasoactive factors released from the endothelium, including endothelin and $\mathrm{NO}^{7}$ Heparin's ability to modulate both the inflammatory response and the elaboration of endothelium-derived vasoactive substance may explain, in part, heparin's protective role during reperfusion injury. It has also recently been demonstrated that heparin protects the myocardium after IR injury through its ability to inhibit the complement cascade. ${ }^{8}$ The ability of heparin to preserve myocardial contractility through the NO-cGMP pathway has not been suggested as a possible protective mechanism of action of heparin during IR injury.

After the identification of endothelium-derived

\section{A}

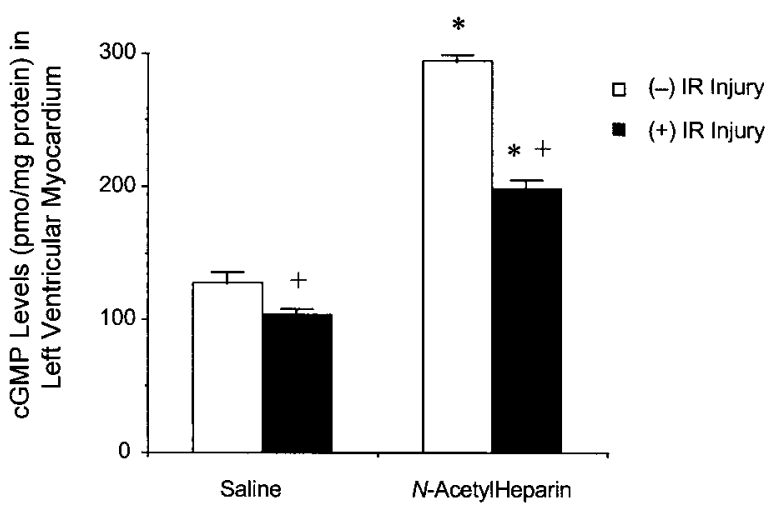

B

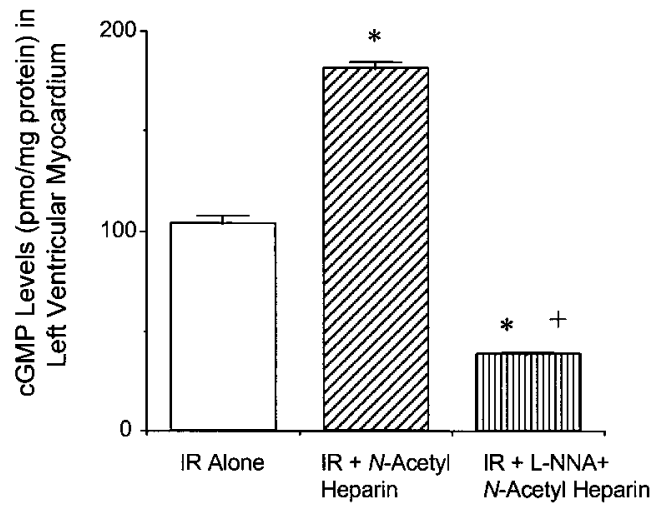

Fig. 3. A, Cyclic guanosine monophosphate (cGMP) levels in left ventricular myocardium after $N$-acetyl heparin treatment and ischemia-reperfusion (IR) injury. Groups represent treatment with saline solution $(n=5), N$-acetyl heparin $(6.0 \mathrm{mg} / \mathrm{kg}$, intravenous, $n=5)$, saline solution + IR injury $(n=5)$, and IR injury $+N$-acetyl heparin $(n=$ $5)$. Data are expressed as means \pm the standard error of the mean. Asterisks indicate $p<0.0001$ for $N$-acetyl heparin versus saline solution in the presence and absence of IR. Asterisk indicates significance for IR injury versus no IR injury in both the saline $(p<0.04)$ and $N$-acetyl heparin treated groups $(p<0.0001)$. B, The group treated with IR injury + L-NNA $+N$-acetyl heparin. Asterisk indicates significance between L-NNA treated group versus IR injury and IR $+N$-acetyl heparin $(p<0.0001)$.

relaxing factor as $\mathrm{NO}^{15}$ research has been performed to identify the physiologic and pathologic roles of NO. Within the vasculature, NO has been identified as a key component in the maintenance of 
Table II. Coagulation parameters before and after treatment with bovine heparin and N-acetylheparin

\begin{tabular}{ccccc}
\hline & Baseline & Bovine heparin & N-Acetyl heparin & $($ Normal $)$ \\
\hline ACT & $87.4 \pm 4.2$ & $780.8 \pm 42.1^{*}$ & $77.6 \pm 11.9$ & $(60-90 \mathrm{sec})$ \\
PTT & $12.4 \pm 0.2$ & $>100^{*}$ & $16.3 \pm 1.4$ & $(10-25 \mathrm{sec})$
\end{tabular}

Values presented as mean \pm standard error of the mean. $A C T$, activated clotting time (sec); PT, prothrombin time (sec); PTT, partial thromboplastin time (sec).

${ }^{*} p \leq 0.001$ compared with baseline values.

vascular tone in the smooth muscle underlying the endothelium, ${ }^{16}$ as well as in the inhibition of smooth muscle cell proliferation. ${ }^{17}$ The role of the endothelium in the paracrine modulation of myocardial function has only recently become an area of extensive study. ${ }^{3}$ Both types of cardiac endothelial cells (coronary vascular and endocardial) regulate myocardial contractility by releasing diffusable vasoactive mediators, such as NO. ${ }^{18}$ Myocardial performance has also been demonstrated to depend on an intact and functional endocardial endothelium. ${ }^{19}$ The role that NO plays in the modulation of myocardial contractility has been studied extensively in different cardiac preparations and in humans. $\mathrm{Nu}-$ merous in vitro studies have demonstrated that NO depresses myocardial contractility in isolated guinea pig cardiac myocytes and in isolated hamster papillary muscles. ${ }^{20,21} \mathrm{NO}$ was also recently shown to attenuate the positive inotropic response to $\beta$-adrenergic stimulation in humans with left ventricular dysfunction. $^{22}$

Despite the well-documented effect of $\mathrm{NO}$ as a negative inotrope, recent studies suggest that endothelium-derived NO and cGMP (the second messenger of NO) in physiologic concentrations, are associated with improved myocardial function and a positive inotropic effect. ${ }^{23,24}$ Mohan and associates $^{23}$ report that the myocardial response to NO and cGMP is a concentration-dependent biphasic contractile response with low physiologic levels stimulating contractility in isolated cat papillary muscles, whereas high pathophysiologic levels result in depressed contractility. These results were confirmed in rat cardiomyocytes exposed to organic nitrates in which moderate increases in cGMP improved the contractile response. ${ }^{24}$ Thus physiologic levels of NO, most likely the result of stimulation of endothelial constitutive NOS (ecNOS), result in a positive inotropic effect, whereas pathophysiologic levels of NO, the result of inducible NOS, classically result in depressed contractility. Inducible NOS, located in macrophages and vascular smooth muscle, is activated by cytokines and endotoxin and requires 6 to 12 hours to be expressed..$^{25}$ Therefore the pathophysiologic levels of NO present in such conditions as cardiomyopathy, cardiac allograft rejection, and septic shock, where myocardial contractility is depressed, are most likely a result of inducible NOS. In this study, the protective effect of heparin and nonanticoagulant heparin is most likely at the level of ecNOS, where physiologic levels of NO and cGMP are beneficial to contractile function. It is unlikely that our pharmacologic intervention is inducing NO given the 2-hour time course of our protocol.

The role of NO in IR injury has also received an enormous amount of attention. NO-mediated vasodilatation has been shown to be diminished after IR injury. ${ }^{26}$ Furthermore, administration of NO donors has been demonstrated to attenuate the deleterious effects of IR injury on both endothelial ${ }^{27}$ and myocardial function. ${ }^{28}$ Polymorphonuclear leukocytes, which are prevalent during reperfusion injury, have been demonstrated to mediate the myocardial contractile dysfunction present during IR injury. ${ }^{29}$ Polymorphonuclear leukocyte-mediated myocardial contractile dysfunction has also been recently demonstrated to be diminished by administration of NO donors. ${ }^{28}$ In this study, inhibition of NOS in the setting of IR injury abolished the protective effect of both heparin and nonanticoagulant heparin during reperfusion injury (Fig. 2). In situations such as IR injury, where the normal homeostatic function of the endothelium is perturbed, diminished levels of both NO and cGMP occur. We have demonstrated that myocardial cGMP levels are significantly diminished during reperfusion injury, confirming the role of $\mathrm{NO}$ in normal myocardial function and its disturbance during IR injury (Fig. 3, $A$ ). Thus it would be logical to postulate that interventions that maintain NO levels may prove beneficial in the maintenance of normal endothelial and myocardial function.

Previous investigators have demonstrated that heparin affords protection to the myocardium in the setting of IR by reducing myocardial necrosis in prolonged models of ischemia ${ }^{8}$ and improving he- 
modynamic factors associated with improved myocardial function. ${ }^{30}$ The data in this study were obtained using an acute episode of IR (15 minutes of ischemia followed by 120 minutes of reperfusion). This protocol better represents the clinical setting of myocardial stunning in the absence of myocardial necrosis. Using this technique, we demonstrated that heparin preserves myocardial contractility by a mechanism independent of its anticoagulant activity. Furthermore, we present a novel mechanism of action of heparin and nonanticoagulant heparin in affording myocardial protection by preserving the NO-cGMP pathway that is critical for normal physiologic function. An alternative pathway of cGMP activation, such as through atrial natiuretic factor stimulation of cGMP, is unlikely given that the heparin-mediated increase in cGMP was abrogated after treatment with L-NNA, indicating that heparin is acting through the NO-cGMP pathway.

In conclusion, $\mathrm{NO}$ is critical for the preservation of normal myocardial function, especially during episodes of IR injury in which the NO-cGMP pathway is perturbed. Furthermore, the administration of heparin and heparin derivatives without anticoagulant properties preserves the NO pathway and prevents myocardial dysfunction after brief episodes of IR. These data may prove clinically significant, allowing the administration of heparin derivatives to preserve myocardial function preventing further injury in the absence of the adverse clinical complications of bleeding.

\section{REFERENCES}

1. Kim YD, Fomsgaard JS, Heim KF, Ramwell PW, Thomas G, Kagan E, et al. Brief ischemia-reperfusion induces stunning of endothelium in canine coronary artery. Circulation 1992; 85:1473-82.

2. Summers ST, Zinner MJ, Freischlag JA. Production of endothelium-derived relaxing factor (EDRF) is compromised after ischemia and reperfusion. Am J Surg 1993;166: 216-20.

3. Paulus WJ, Vantrimpont PJ, Shah AM. Paracrine coronary endothelial control of left ventricular function in humans. Circulation 1995;92:2119-26.

4. Mohan P, Sys SU, Brutsaert DL. Positive inotropic effect of nitric oxide in myocardium. Int J Cardiol 1995;50:233-7.

5. Hasebe N, Shen YT, Vatner SF. Inhibition of endotheliumderived relaxing factor enhances myocardial stunning in conscious dogs. Circulation. 1993;88:2862-71.

6. Saliba MJ Jr, Covell JW, Bloor CM. Effects of heparin in large doses on the extent of myocardial ischemia after acute coronary occlusion in the dog. Am J Cardiol 1976;37:599-604.

7. Yokokawa, K, Tahara H, Kohno M, Mandal AK, Yanagisawa M, Takeda T. Heparin regulates endothelin production through endothelium-derived nitric oxide in human endothelial cells. J Clin Invest 1993;92:2080-5.
8. Black SC, Gralinski MR, Friedrichs GS, Kilgore KS, Driscoll EM, Lucchesi BR. Cardioprotective effects of heparin or $N$-acetyl heparin in an in vivo model of myocardial ischaemic and reperfusion injury. Cardiovasc Res 1995;29:629-36.

9. Theroux P, Franklin D, Ross J Jr, Kemper WS. Regional myocardial function during acute coronary artery occlusion and its modification by pharmacologic agents in the dog. Circ Res 1974;35:896-908.

10. Duncker DJ, Bache RJ. Inhibition of nitric oxide production aggravates myocardial hypoperfusion during exercise in the presence of a coronary artery stenosis. Circ Res 1994;74:62940.

11. Cailla HL, Vannier CJ, Delaage MA. Guanosine $3^{\prime} 5^{\prime}$-cyclic monophosphate assay at the $10^{-15}$ mole level. Anal Biochem 1976;70:195-202.

12. Rosenberg RD. The heparin-antithrombin system: a natural anticoagulant mechanism. In: Colman RW, Hirsh J, Marder VJ, Salzman EW, editors. Hemostasis and thrombosis: basic principles and clinical practice. Philadelphia: JB Lippincott; 1987. p. 1373-92.

13. Pasini FL, Pasqui AL, Ceccetalli L, Capecchi PL, Orrico A, DiPerri T. Heparin inhibition of PMN activation in vitro: a possible pharmacological approach to granulocyte mediated vascular damage. Thromb Res 1984;35:527-37.

14. Kazatchkine MD, Fearon DT, Metcalfe DD, Rosenberg RD, Austen KF. Structural determinants of the capacity of heparin to inhibit the formation of the human amplification C3 convertase. J Clin Invest 1981;67:223-8.

15. Palmer RMJ, Ferrige AG, Moncada S. Nitric oxide release accounts for the biologic activity of endothelium-derived relaxing factor. Nature 1987;327:524-6.

16. Furchgott RF. Role of endothelium in responses of vascular smooth muscle. Circ Res 1983;53:557-73.

17. Garg UC, Hassid A. Nitric oxide-generating vasodilators and 8-bromo-cyclic guanosine monophosphate inhibit mitogenesis and proliferation of cultured rat vascular smooth muscle cells. J Clin Invest 1989;83:1774-7.

18. Lawson DL, Mehta JL, Nichols WW. 1990. Coronary reperfusion in dogs inhibits endothelium-dependent relaxation: role of superoxide radicals. Free Radic Biol Med 1990;8:37380.

19. Dignan RJ, Dyke CM, Abd-Elfattah AS, Lutz HA, Yeh T Jr, Lee KF, et al. Coronary artery endothelial cell and smooth muscle dysfunction after global myocardial ischemia. Ann Thorac Surg 1992;53:311-7.

20. Brady AJ, Warren JB, Poole-Wilson PA, Williams TJ, Harding SE. Nitric oxide attenuates cardiac myocyte contraction. Am J Physiol 1993;265:H176-82.

21. Finkel MS, Oddis CV, Mayer OH, Hattler BG, Simmons RL. Nitric oxide synthase inhibitor alters papillary muscle forcefrequency relationship. J Pharmacol Exp Ther 1995;272:94552.

22. Hare JM, Loh E, Creager MA, Colucci WS. Nitric oxide inhibits the positive inotropic response to $\beta$-adrenergic stimulation in humans with left ventricular dysfunction. Circulation 1995;92:2198-203.

23. Mohan P, Brutsaert DL, Paulus WJ, Sys SU. Myocardial contractile response to nitric oxide and cGMP. Circulation 1996;93:1223-9

24. Kojda G, Kottenberg K, Nix P, Schluter KD, Piper HM, Noack E. Low increase in cGMP induced by organic nitrates 
and nitrovasodilators improves contractile response of rat ventricular myocytes. Circ Res 1996;78:91-101.

25. Balligand JL, Kelly RA, Marsden PA, Smith TW, Michel T. Control of cardiac muscle cell function by an endogenous nitric oxide signaling system. Proc Natl Acad Sci U S A 1993; 90:347-51.

26. Sternbergh WC III, Makhoul RG, Adelman B. Nitric oxidemediated, endothelium-dependent vasodilation is selectively attenuated in the postischemic extremity. Surgery 1993;114: 960-7.

27. Siegfried MR, Erhardt J, Rider T, Ma XL, Lefer AM. Cardioprotection and attenuation of endothelial dysfunction by organic nitric oxide donors in myocardial ischemia-reperfusion. J Pharmacol Exp Ther 1992;260:668-75.

28. Pabla R, Buda AJ, Flynn DM, Blesse SA, Shin AM, Curtis $\mathrm{MJ}$, et al. Nitric oxide attenuates neutrophil-mediated myocardial contractile dysfunction after ischemia and reperfusion. Circ Res 1996;78:65-72.

29. Engler R, Covell JW. Granulocytes cause reperfusion ventricular dysfunction after 15-minute ischemia in the dog. Circ Res 1987;61:20-8.

30. Friedrichs GS, Kilgore KS, Manley PJ, Gralinski MR, Lucchesi BR. Effects of heparin and $N$-acetyl heparin on ischemia/reperfusion-induced alterations in myocardial function in the rabbit isolated heart. Circ Res 1994;75:701-10.

\section{Discussion}

Dr. Pedro J. del Nido (Boston, Mass.). My first question relates to whether you are looking at NO effects on coronary vasculature, that is improved reperfusion, or are you looking at direct myocardial suppressant effect of NO? Most of us think of the latter being dependent on very high concentrations of $\mathrm{NO}$ and is that in fact what you are generating with these heparin derivatives?

Dr. Kouretas. First of all, I think it is both. I think it is an effect of heparin on the endothelium-derived NO and I also think it is an effect of heparin on myocardial levels of NO. An enormous amount of literature suggests that NO depresses myocardial contractility. However, after carefully reviewing the literature, the levels of NO generated in those studies were very high, most likely resulting from inducible NO. In our studies, we do not think that we are dealing with inducible NO because our protocol is a short period of ischemia (15 minutes) and reperfusion (120 minutes). Therefore I do not think inducible NO is at play because that takes 6 hours to be induced. We are most likely observing the effect of constitutive venoarterial NO.

Recent studies also indicate that physiologic levels of NO elaborated by constitutive NO actually produce a positive inotropic effect and not a negative inotropic effect. Therefore we think that the positive inotropic effect could be at two levels, both at the endothelial level of the vasculature and at a direct myocardial level.

Dr. del Nido. And that leads to my follow-up question. If, in fact, the heparin derivatives are increasing cGMP production, can you give too much of this? In other words, can you generate such a large amount of NO with these medications that you actually lead to myocardial suppression?

Dr. Kouretas. First of all, I think to generate too much NO you would probably have to induce NO. Heparin in and of itself I do not think would cause that much NO to be released. Therefore I think heparin is acting more at a physiologic level (constitutive NO) than at pathophysiologic levels (inducible NO).

Dr. Frank W. Sellke (Boston, Mass.). It has been known that heparin causes a release of endothelium-derived NO. And in addition, other agents that cause the release of NO, such as sodium nitroprusside and nitroglycerin, have similar beneficial effects. Do you think this is a specific effect of heparin or can it be expanded to any agent that releases NO?

Second, heparin is a very potent inhibitor of complement activation. So there may be both an indirect effect of myocardial preservation through the release of NO or a direct effect by inhibition of complement activation.

Dr. Kouretas. The fact that so many studies have shown that NO is beneficial in the setting of IR injury. I think the fact that heparin releases NO is only one aspect of this complicated puzzle. Heparin also has other properties changing the variations of complement and as an "antiinflammatory" agent. Studies have also shown that inflammation is a very important component in reperfusion injury. So I think heparin can be acting at several levels, at the level of NO, which is one area that we focused on, but I think it is functioning at many levels. For example, inhibiting inflammation in the setting of reperfusion injury would be protective. It has also been shown to be a potent antioxidant, free-radical scavenger, which is also important in reperfusion injuries. So I think heparin can be working on many levels and not just on NO. That is just the area that we focused on.

Dr. John E. Mayer, Jr. (Boston, Mass.). As you probably know, we have been interested in similar sorts of areas for some time. As an additional way to confirm the role of NO, have you thought about using L-arginine or something else that would increase endothelial production of NO to see whether you can, in fact, offset the effect of your NOS inhibitor?

Dr. Kouretas. We have not done those experiments. That is a good suggestion to see whether we can replenish endothelial function in the setting of reperfusion, but we have not completed those studies at this time.

Dr. Brian Buxton (Heidelberg, Victoria, Australia). Protamine reverses the anticoagulant effect of heparin. Does protamine abolish the preservation by nonanticoagulant heparin of regional myocardial contractility after IR injury? If so, this may explain at least in part the finding of myocardial depression of protamine administration after cardiopulmonary bypass.

Dr. Kouretas. A lot of investigators have speculated that the effect of heparin is based on its highly negatively charged structure. So one can speculate that protamine, which is highly positively charged, might abrogate the protective effect of heparin. On the flip side, other investigators have shown that protamine also releases NO. So I think it is a very complicated question and needs to be addressed.

Dr. Edward D. Verrier (Seattle, Wash.). I think the other thing that is interesting is that NO really can be a double-edged sword in IR. It would be very interesting to see the inducible form of NO, which is up-regulated by ischemia. Expression would take 4 to 6 hours to complete 
because controversy exists in the literature concerning the efficacy of inducible $\mathrm{NO}$ with ischemia. It would be interesting to see what would happen at 6 hours or 8 hours to infarct size. The results may be different from the conclusions of this presentation.

Dr. Gregory M. Hirsch (Halifax, Nova Scotia, Canada). Some years ago I investigated heparin fragments for their antiproliferative activity and in fact used various combinations of fragment length and degree of carbon acetylation. I wonder whether you might comment on either restrictions of size for the maintenance of this effect and particularly describe the fragment and degree of acetylation that you used.

Dr. Kouretas. (Slide) The structure of heparin is one of a repeating disaccharide unit. The structure of $N$-acetyl heparin, the nonanticoagulant heparin derivative, has an acetyl group placed instead of sulfate, which inhibits its binding to antithrombin III. So basically this fraction of heparin is the same length as regular bovine heparin with only the substitution of this acetyl group. So this fraction is actually the same length of bovine heparin that causes the anticoagulant properties.

\section{Bound volumes available to subscribers}

Bound volumes of The Journal of Thoracic and Cardiovascular Surgery are available to subscribers (only) for the 1998 issues from the Publisher, at a cost of $\$ 122.00$ for domestic, $\$ 151.94$ for Canadian, and $\$ 142.00$ for international subscribers for Vol. 115 (January-June) and Vol. 116 (July-December). Shipping charges are included. Each bound volume contains a subject and author index and all advertising is removed. Copies are shipped within 60 days after publication of the last issue of the volume. The binding is durable buckram with the Journal name, volume number, and year stamped in gold on the spine. Payment must accompany all orders. Contact Mosby, Inc., Subscription Services, 11830 Westline Industrial Drive, St. Louis, Missouri 63146-3318, USA; phone 800-453-4351 or 314-453-4351.

Subscriptions must be in force to qualify. Bound volumes are not available in place of a regular Journal subscription. 